\title{
Effect of memory set size on visual evoked potential for digit and figure stimuli
}

\author{
MARIKO OSAKA \\ Kyoto University, Kyoto, Japan \\ and \\ NAOYUKI OSAKA \\ Otemon-Gakuin University, Osaka, Japan
}

\begin{abstract}
Visual evoked potential and reaction time (RT) were recorded during the performance of memory retrieval task of Sternberg's paradigm. Ten subjects received a series of memory set of $1,3,5$, and 7 digits, and another 10 subjects received memory sets $1,3,5$, and 7 figures. EEGs were recorded from $\mathrm{Cz}$ to obtain visual evoked potentials to the test stimuli. P300 and RT latencies increased linearly with increasing memory set size, but the slope for P300 was less than that for RT. The differences between the P300 slope and the RT slope were larger for the figure condition, which was more difficult than the digit condition due to the increasing difficulty of sequential pattern matching in memory.
\end{abstract}

In a short-term-memory experiment, it is known that reaction time (RT) increases linearly with increasing memory set size, which indicates a sequential and exhaustive memory search (Sternberg, 1966).

Recent investigations on visual evoked potential (VEP) have indicated that $\mathrm{P} 300$ reflects stimulus evaluation relevant to task performance and that the latency of P300 changes as a function of time required for stimulus evaluation. Several investigators (Adam \& Collins, 1978; Ford, Roth, Mohs, Hopkins, \& Kopell, 1979; Roth, Kopell, Tinklenberg, Darley, Sikora, \& Vesecky, 1975) recorded VEP during the performance of memory retrieval tasks and reported that the latency of the P300 component of VEP increased as a function of memory set size. There have been few studies, however, that have used a figure-matching-performance paradigm. It is likely that the time elapsing between P300 and RT is longer due to the low confidence associated with the more difficult task (Ford et al., 1979). In the present experiment, VEP and RT were recorded during performance on Sternberg's (1966) paradigm in an attempt to investigate the stimulus effects of digits and figures.

\section{METHOD}

\section{Subjects}

Twenty subjects (18 to 25 years old) participated in the experiment. Ten subjects received a series of memory sets of 1,3 , 5 , and 7 digits. Another 10 subjects received a series of similarly sized sets of figures.

This work was supported by Grants 57710045 and 58710057 , from the Ministry of Education of Japan to the first author. Address reprint requests to: Mariko Osaka, Department of Educational Psychology, Faculty of Education, Kyoto University, Kyoto 606, Japan.

\section{Procedure}

The subjects were instructed to press the microswitch key to the right or to the left, depending on whether the test digit was or was not a member of the memory set. Of each of the four set sizes, $50 \%$ included the test stimulus in the memory set and $50 \%$ did not. The experimental session lasted until 24 correct "yes" responses had been made. The digits (figures) were presented successively, for $250 \mathrm{msec}$ each, with a 500 -msec interstimulus interval. One second after memory set presentation had concluded, a 250-msec warning stimulus came on. One second after the warning stimulus had come on, a test digit appeared and remained on for 1 sec. The stimulus was generated by a computer CRT (Commodore PET-2001 microcomputer) and presented on a video monitor screen (P31 phosphor) in a darkened room. The stimuli were presented within an area $1 \mathrm{~cm}$ square at eye level at a distance of $1 \mathrm{~m}$ from the subject. The EEG was recorded from $\mathrm{Cz}$ (according to the 10/20 international electrode system) with the right ear lobe as reference; Beckman silver/silver-chloride disc electrodes were used. The EOG was recorded from electrodes above and below the right eye to detect artifacts produced by eye blinks. The signals were amplified by an EEG amplifier, with a time constant of $1.5 \mathrm{sec}$. Averaging was done off-line on a signal processor (San'ei 7T08 averaging processor) with an appropriate averaging program. Averaging began $64 \mathrm{msec}$ before the test digit had appeared and continued for $960 \mathrm{msec}$ after the test digit had disappeared. Sampling rate was 1,000 samples/sec $(1 \mathrm{sample} / \mathrm{m} \mathrm{sec})$. The VEPs were averaged when the test stimulus was in the memory set and the response had been correct. Any RT longer than 1,500 msec after the presentation of test digit was categorized as "no response." The average was based on 24 trials for each set size. The subjects were instructed to respond as quickly as they could while trying to avoid errors.

\section{RESULTS AND DISCUSSION}

Figure 1 shows the mean RTs across subjects for digit and figure stimuli. RTs increase as the memory set size

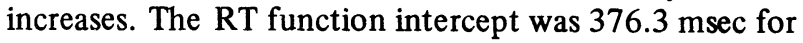




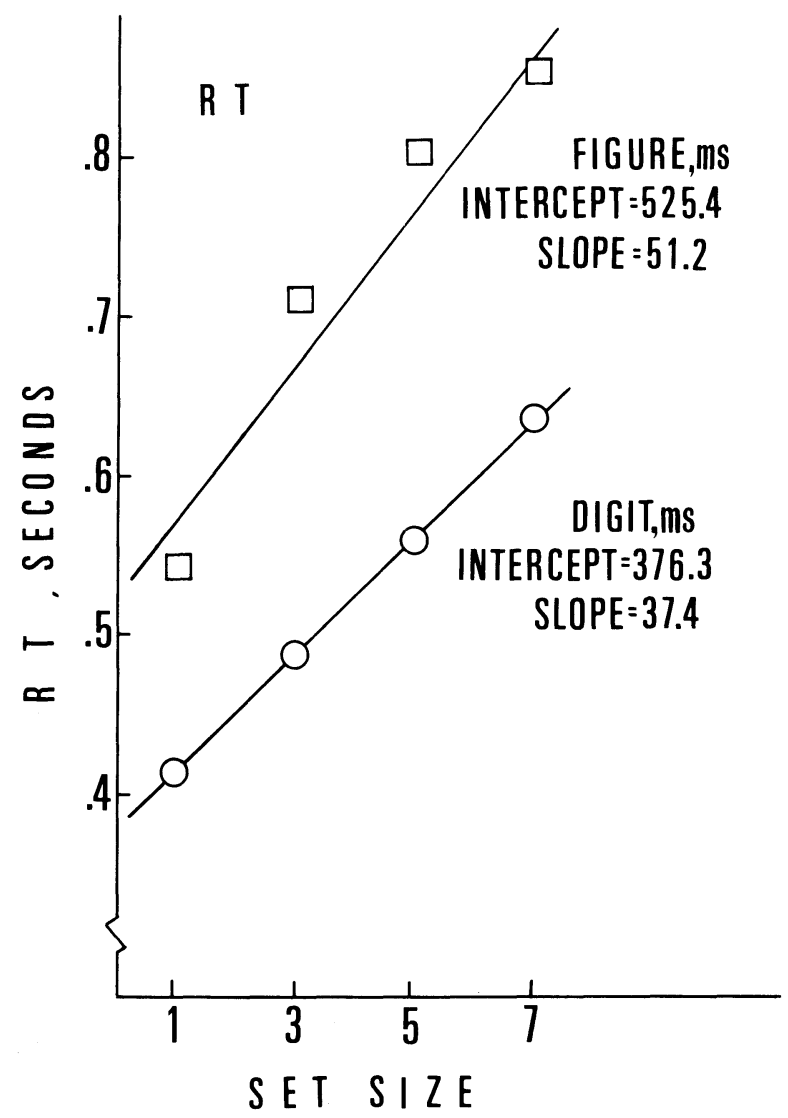

Figure 1. Mean RT as a function of memory set size for digit and figure stimuli.

the digit condition and was $525.4 \mathrm{msec}$ for the figure condition. RT increased approximately $37.4 \mathrm{msec}$ for each additional memory set item in the digit condition, whereas RT increased about $51.2 \mathrm{msec}$ per item in the figure condition. An analysis of variance (ANOVA) indicated that RT increased with increasing memory set size $[F(3,54)=156.28, p<.001]$. RT was slower for figure stimuli than for digit stimuli $[\mathrm{F}(1,18)=29.76$, $\mathrm{p}<.001]$. Moreover, the Stimulus (digit vs. figure) by Set Size interaction was statistically significant $[F(3,54)$ $=7.97, \mathrm{p}<.01]$. The increase in RT with increasing memory set size differed for the digit and figure conditions. For each memory set in the figure condition, more time was needed for searching for one item.

Figure 2 presents the averaged $\mathrm{P} 300$ latencies across subjects for digit and figure stimuli as a function of memory set size. P300 to the test stimulus was measured as the maximum positive peak occurring between 250 and $800 \mathrm{msec}$. An ANOVA indicated that the P300 latencies increased as a function of memory set size $[F(3,54)=84.59, p<.001]$. And P300 latency was greater for figure stimuli than for digit stimuli $[\mathrm{F}(1,18)$ $=9.77, \mathrm{p}<.001]$. The P300-latency-function intercept was $338.7 \mathrm{msec}$ for the digit condition and $381.0 \mathrm{msec}$ for the figure condition. The slopes of the P300 latency function were 26.5 and $36.8 \mathrm{msec}$ for the digit condition and the figure condition, respectively. The interaction of Stimulus by Set Size was marginally significant $[F(3,54)$ $=2.73, \mathrm{p}<.053]$. These results indicate that P300 latency to the test stimulus increases linearly as a function of memory set size. This finding is in agreement with results reported by other investigators: Adam and Collins (1978) and Ford et al. (1979) found the slope values for digits to be approximately 22 and $27 \mathrm{msec}$, respectively, and the slope value for the P300 latency function in this experiment was about $26 \mathrm{msec}$ for digits.

In the present experiment, the slope difference between RT and P300 latency function was about $10 \mathrm{msec}$ for the digit stimuli, whereas, for the figure stimuli, the difference was about 14 msec. ANOVA indicated that the RT-P300 latency value increased with increasing memory set size $[F(3,54)=13.89, p<.01]$. Moreover, the RT-P300 latency value was larger for figure stimuli than for digit stimuli $[\mathrm{F}(1,18)=8.10, \mathrm{p}<.001]$. This suggests that the time elapsing between P300 and RT becomes larger with increasing set size. Furthermore, delay time was greater for figure stimuli than for digit stimuli. It is likely that the figure condition was more difficult than the digit condition due to difficulty of phonetic retrieval, and this may have caused significant differences. Ford et al. (1979) mentioned that slope dif-

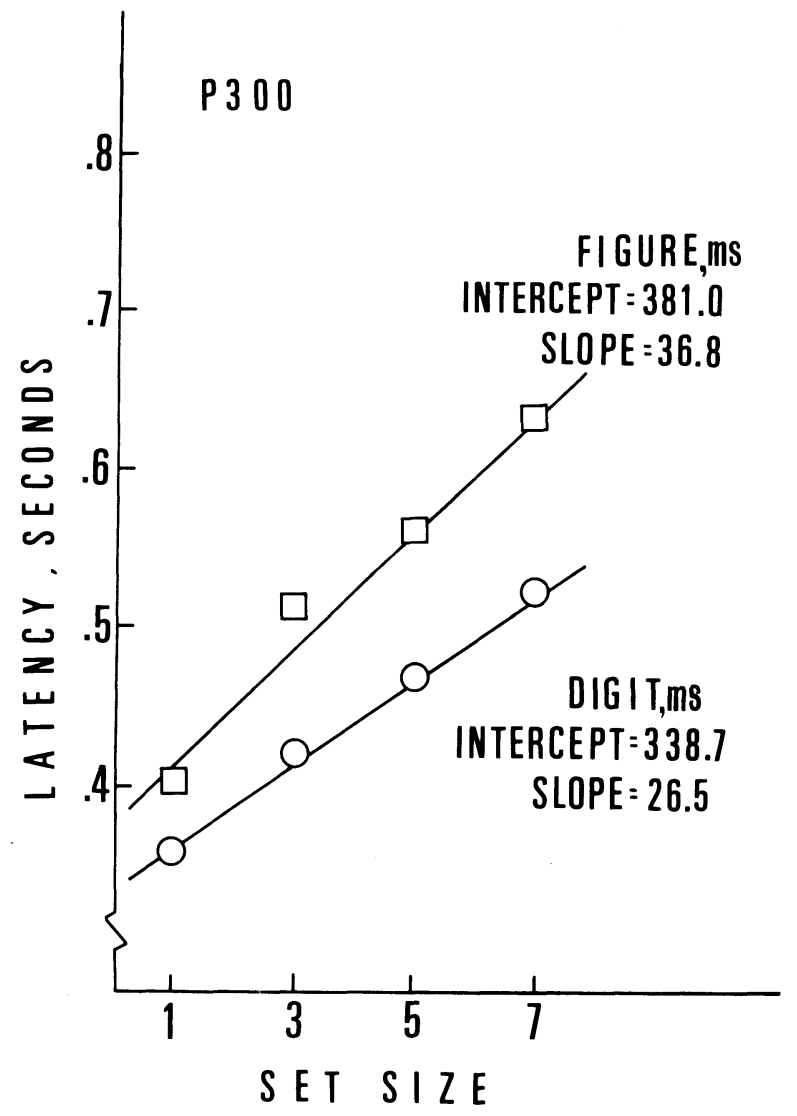

Figure 2. Averaged P300 latency as a function of memory set size for digit and figure stimuli. 
ference is affected by level of confidence, taking longer when the subject is less confident. The present results indicate that the subjects were less confident of the accuracy of their responses in the figure condition than in the digit condition (Osaka \& Osaka, Note 1).

The RT intercept was larger than that for P300 latency both for digit and for figure stimuli. Moreover, the intercept difference was larger for figure stimuli (144.4 msec) than for digit stimuli $(37.6 \mathrm{msec})$.

According to the present results, it appears that P300 and RT occur at about the same time when P300 is based on underlying processes that have a close relationship to task-relevant decision making and response selection, whereas, the time delay between P300 and RT becomes larger when the stimulus evaluation of P300 is less relevant to decision making, due to increasing difficulty of sequential pattern matching in memory.

\section{REFERENCE NOTE}

1. Osaka, M., \& Osaka, N. Effect of memory set size on visual evoked potential. Paper to be presented at the annual meeting of the American Psychological Association, Anaheim, California, August 1983.

\section{REFERENCES}

Adam, N., \& Collins, G. I. Late components of the visual evoked potential to search in short-term memory. Electroencephalography and Clinical Neurophysiology, 1978, 44, 147-156.

Ford, J. M., Roth, W. T., Mors, R. C., Hopkins, W. F., \& Kopell, B. S. Event-related potentials recorded from young and old adults during a memory retrieval task. Electroencephalography and Clinical Neurophysiology, 1979, 47, 450-459.

Roth, W. T., Kopell, B. S., Tinklenberg, J. R., Darley, C. F., Sikora, R., \& Vesecky, T. B. The contingent negative variation during a memory retrieval task. Electroencephalography and Clinical Neurophysiology, 1975, 38, 171-174.

Sternberg, S. High-speed scanning in human memory. Science, $1966,153,652-654$.

(Received for publication April 18, 1983.) 